\title{
Evaluation of serum iron levels during FOLFOX4 and FOLFIRI therapies
}

\author{
SATOMI MASHIKO ${ }^{1}$, ISAO NAGAOKA ${ }^{3}$, MASAYUKI KITAJIMA ${ }^{2}$, TOMOO WATANABE $^{2}$, \\ KAZUHIKO NISHIMURA ${ }^{2}$, SHUNJI FUTAGAWA ${ }^{2}$ and TAKUMI OCHIAI ${ }^{2}$ \\ Departments of ${ }^{1}$ Pharmacy, and ${ }^{2}$ Surgery, Tobu Chiiki Hospital, Tokyo Metropolitan Health and \\ Medical Treatment Corporation, Tokyo; ${ }^{3}$ Department of Host Defense and Biochemical Research, \\ Juntendo University School of Medicine, Tokyo, Japan
}

Received January 27, 2010; Accepted March 22, 2010

DOI: $10.3892 /$ etm_00000080

\begin{abstract}
FOLFOX4 and FOLFIRI are effective regimens for the treatment of advanced colorectal cancer, and their use together with molecular targeting drugs has recently become more common. In the present study, we evaluated the changes in the serum iron levels of patients undergoing FOLFOX4 or FOLFIRI therapy alone or in combination with bevacizumab (BV). The serum iron level was increased $48 \mathrm{~h}$ after therapy and was restored to baseline 2 weeks afterwards in colorectal cancer patients who received FOLFOX4 or FOLFIRI alone or in combination with BV. This transient increase in serum iron was observed repeatedly during chemotherapy. The serum iron level was $71.66 \pm 28.96 \mu \mathrm{g} / \mathrm{dl}$ (mean \pm standard deviation) before treatment and significantly increased to $186.82 \pm 83.17 \mu \mathrm{g} / \mathrm{dl}(\mathrm{p}<0.001) 48 \mathrm{~h}$ after therapy. A transient increase in serum iron levels was also observed when FOLFIRI was administered to a patient after tumor resection. In contrast, no decrease in blood hemoglobin, no increase in liver enzymes and no increase in urinary iron excretion were observed. Based on these results, it can be concluded that an increase in serum iron may be induced by a transient change in iron distribution within the body after FOLFOX4/FOLFIRI therapy with or without BV.
\end{abstract}

\section{Introduction}

FOLFOX4 therapy [folinic acid (FOL), fluorouracil (F) plus oxaliplatin (OX)] (1) and FOLFIRI therapy (FOL, F, plus irinotecan (IRI)] (2) are international standard treatments for advanced colorectal cancer (3). In recent years, it has also

Correspondence to: Dr Takumi Ochiai, Department of Surgery, Tobu Chiiki Hospital, Tokyo Metropolitan Health and Medical Treatment Corporation, 5-14-1 Kameari, Katsushika, Tokyo 125-8512, Japan

E-mail: takumi-o@ma.kitanet.ne.jp

Key words: serum iron, chemotherapy, FOLFOX4, FOLFIRI, colorectal cancer been recommended that molecular targeting drugs such as bevacizumab $(\mathrm{BV})(4,5)$ or cetuximab (Cet) $(6,7)$ be combined with FOLFOX4 or FOLFIRI.

The median patient survival time (MST) was reported to be significantly longer for FOLFOX4 + BV therapy (12.9 months) than for FOLFOX4 alone (10.8 months), confirming that the addition of BV increased the efficacy of the treament (4). The MST was also reported to be significantly longer for FOLFIRI + Cet (8.9 months) than for FOLFIRI alone (8.0 months) (8). These observations suggest that chemotherapy combined with molecular targeting drugs is more effective for treatment of advanced colorectal cancer. Thus, the addition of such molecular targeting drugs to chemotherapy has been recommended (Saltz LB, et al: Proc ASCO 170: abs. 4028, 2007) .

Anemia is one of the most common adverse effects of chemotherapy. However, Follézou et al (9) reported an increase in the serum iron level after the administration of various anticancer drugs, including 5-FU, actinomycin D, adriacin and cyclophosphamide. Yet, there have been no reports concerning the effect of FOLFOX4 and FOLFIRI on serum iron levels. Therefore, in the present study, we evaluated the effect of FOLFOX4 and FOLFIRI therapies on changes in serum levels of iron as well as transferrin and ferritin.

\section{Materials and methods}

Subjects. Fifty-eight subjects (92 cases) were enrolled in this study. They were admitted to Tobu Chiiki Hospital (Tokyo Metropolitan Health and Medical Treatment Corporation, Tokyo, Japan) and received FOLFOX4 or FOLFIRI therapy alone or in combination with BV between April 2005 and September 2008. Prior to the enrollment, informed consent was obtained from all the subjects. The patient characteristics are presented in Table I.

Measurement of the serum iron level. Serum iron was measured by the hospital laboratory before and $48 \mathrm{~h}$ after treatment in the 44 patients receiving FOLFOX4 therapy. The normal range of serum iron was $60-210 \mu \mathrm{g} / \mathrm{dl}$ for men and $50-170 \mu \mathrm{g} / \mathrm{dl}$ for women. The serum iron level was also measured before and after treatment in the 11 patients receiving FOLFOX $4+\mathrm{BV}$. Furthermore, serum iron levels were compared before and 
Table I. Characteristics of the subjects.

\begin{tabular}{lc}
\hline No. of subjects & 58 \\
Gender (male/female) & $35 / 23$ \\
Mean age (years) & $69.4 \pm 7.7$ \\
Dukes' stage (A/B/C/D) & $1 / 9 / 28 / 20$ \\
Colon/rectum & $37 / 21$ \\
Recurrence & \\
Liver & 23 \\
Lung & 9 \\
Peritoneum & 7 \\
Lymph node & 5 \\
Local recurrence & 5 \\
Bone & 1 \\
Brain & 1 \\
Other & 3 \\
Unresectable & 4 \\
\hline
\end{tabular}

after the introduction of $\mathrm{BV}$ in the 10 patients who received FOLFOX4 + BV after FOLFOX4 alone.

Serum iron was measured before and after treatment in the 31 patients who received FOLFIRI therapy, and in the 6 patients who received FOLFIRI + BV. The serum iron level was also compared before and after the introduction of $\mathrm{BV}$ in the 5 patients who received FOLFIRI + BV after FOLFIRI alone.

Measurement of transferrin and ferritin. Transferrin and ferritin levels were measured before and after treatment at SRL, Inc. (Tokyo, Japan) in the 15 and 14 patients who received FOLFOX4 and FOLFIRI therapy, respectively. The normal range of transferrin was $190-300 \mathrm{mg} / \mathrm{dl}$ for men and 200-340 mg/dl for women, while the normal range of ferritin was 39.4-340 ng/ml for men and 3.6-114 ng/ml for women.

Measurement of urinary iron. Urinary iron was measured at the hospital laboratory on the day of treatment and on the next day in 5 and 7 patients who received FOLFOX4 and FOLFIRI therapy, respectively.

Statistical analysis. The t-test was used to compare the two groups, and $\mathrm{p}<0.05$ was considered to be significant. Data are expressed as the mean \pm standard deviation (SD).

\section{Results}

Changes in serum iron levels during FOLFOX4 therapy. A typical pattern of the changes in the serum iron levels before and after FOLFOX4 therapy is shown in Fig. 1. The serum iron level transiently increased after treatment $(48 \mathrm{~h})$ and then returned to baseline within 2 weeks. In the FOLFOX 4 group (44 patients and 272 blood samples), the serum iron level was $68.24 \pm 25.20 \mu \mathrm{g} / \mathrm{dl}$ before treatment and increased significantly to $143.34 \pm 62.18 \mu \mathrm{g} / \mathrm{dl}$ afterwards ( $<<0.001$, Fig. 2), showing an increase of $238.54 \pm 127.17 \%$. In the FOLFOX4 + BV group, the serum iron level also increased transiently after treatment (48 h), and then returned to baseline within 2 weeks (data not shown). In the FOLFOX4 + BV group (11 patients and 46 blood

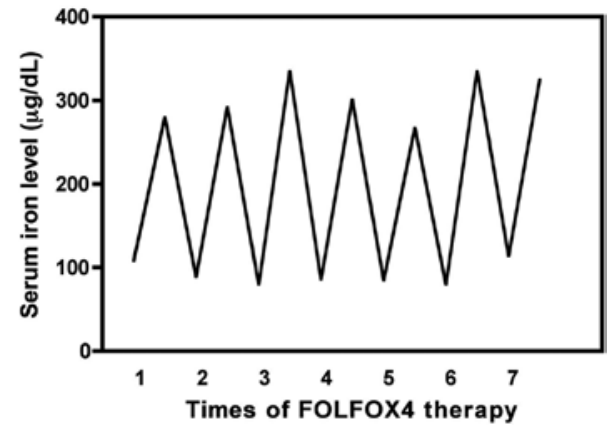

Figure 1. A typical pattern of the changes in the serum iron levels before and after FOLFOX4 therapy.

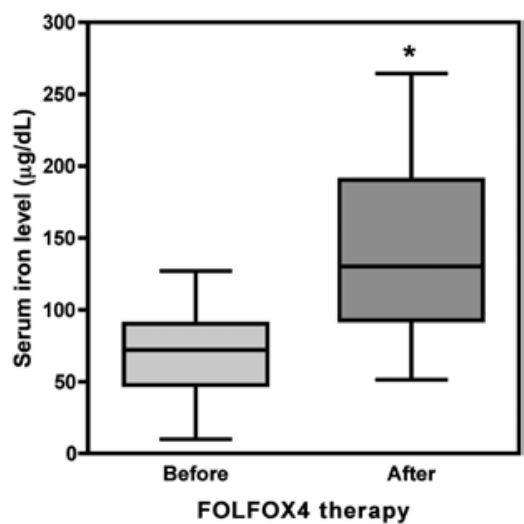

Figure 2. Changes in the serum iron levels before and after FOLFOX4 therapy. Serum iron levels were measured using 272 blood samples from 44 patients. Data are the mean $\pm \mathrm{SD}$, and values are compared before and after FOLFOX4 therapy. ${ }^{*} \mathrm{p}<0.001$.

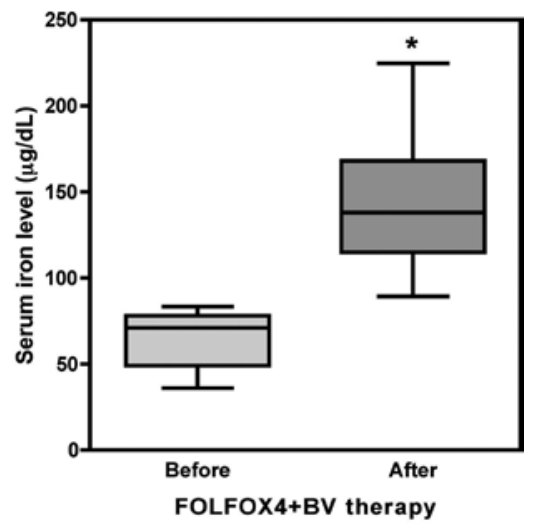

Figure 3. Changes in the serum iron levels before and after FOLFOX $4+$ BV therapy. Serum iron levels were measured using 46 blood samples from 11 patients. Data are the mean $\pm \mathrm{SD}$, and values are compared before and after FOLFOX4 + BV therapy. "p $<0.001$.

samples), the serum iron level was $65.59 \pm 15.87 \mu \mathrm{g} / \mathrm{dl}$ before treatment and increased significantly to $147.55 \pm 44.55 \mu \mathrm{g} /$ dl after treatment $(\mathrm{p}<0.001$, Fig. 3), showing an increase of $247.16 \pm 60.70 \%$.

Changes in serum iron levels during FOLFIRI therapy. A typical pattern of the changes in the serum iron levels before 


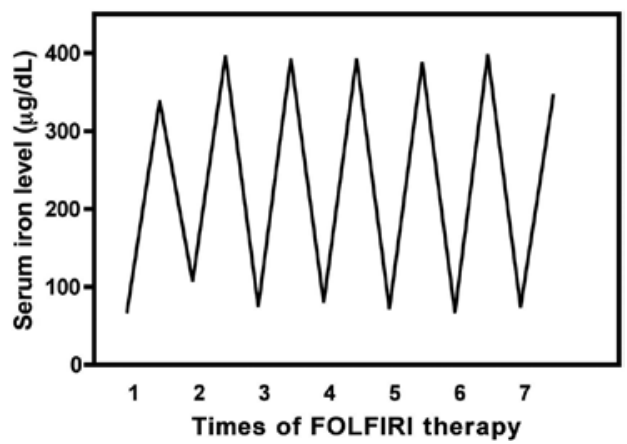

Figure 4. A typical pattern of the changes in the serum iron levels before and after FOLFIRI therapy.

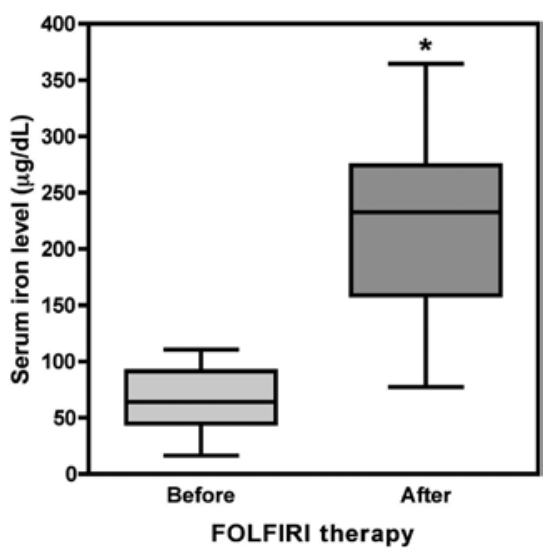

Figure 5. Changes in the serum iron levels before and after FOLFIRI therapy. Serum iron levels were measured using 231 blood samples from 31 patients. Data are the mean $\pm \mathrm{SD}$, and values are compared before and after FOLFIRI therapy. " $\mathrm{p}<0.001$.

and after FOLFIRI therapy is shown in Fig. 4. The serum iron level transiently increased after treatment $(48 \mathrm{~h})$ and then returned to baseline within 2 weeks. In the FOLFIRI group (31 patients and 231 blood samples), the serum iron level was $66.01 \pm 27.47 \mu \mathrm{g} / \mathrm{dl}$ before treatment and increased significantly to $221.69 \pm 78.51 \mu \mathrm{g} / \mathrm{dl}$ afterwards ( $<<0.001$, Fig. 5), showing an increase of $399.94 \pm 6.25 \%$. In the FOLFIRI + BV group, the serum iron level also increased transiently after treatment $(48 \mathrm{~h})$ and then returned to baseline within 2 weeks (data not shown). In the FOLFIRI + BV group (6 patients and 26 blood samples), the serum iron level was $64.68 \pm 23.60 \mu \mathrm{g} / \mathrm{dl}$ before treatment and increased significantly to $244.55 \pm 40.54 \mu \mathrm{g} /$ dl after treatment $(\mathrm{p}<0.001$, Fig. 6), showing an increase of $440.33 \pm 156.22 \%$.

Since there was little difference in the changes in serum iron between FOLFOX 4 and FOLFIRI therapy when these regimens were combined with BV (Figs. 2, 3, 5 and 6), BV was considered to impart no influence on the changes in iron levels. To confirm this, changes in serum iron were examined in patients who underwent FOLFOX4 + BV after FOLFOX4 alone. No difference was noted in the serum iron levels after treatment between FOLFOX4 alone $(80.84 \pm 53.94 \mu \mathrm{g} / \mathrm{dl}, \mathrm{n}=10)$ and FOLFOX $4+$ BV $(76.03 \pm 34.84 \mu \mathrm{g} / \mathrm{dl})$. Furthermore, the serum iron levels were also measured in patients who under-

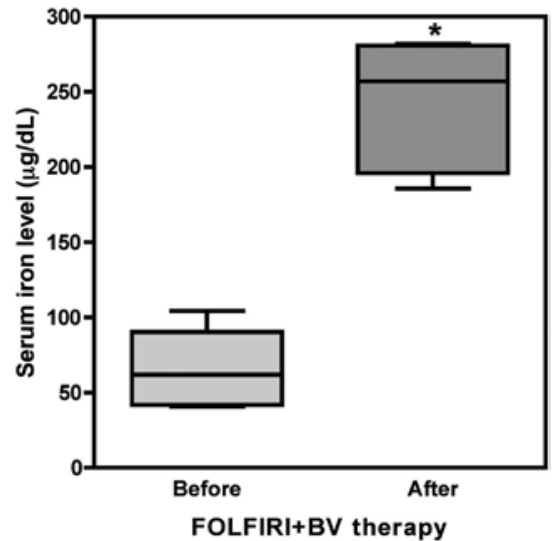

Figure 6. Changes in the serum iron levels before and after FOLFIRI + BV therapy. Serum iron levels were measured using 26 blood samples from 6 patients. Data are the mean $\pm \mathrm{SD}$, and values are compared before and after FOLFIRI therapy. * $\mathrm{p}<0.001$.

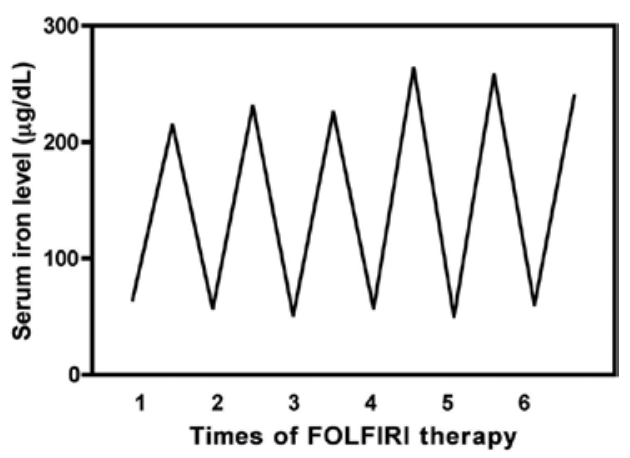

Figure 7. A typical pattern of the changes in the serum iron levels before and after FOLFIRI therapy in a tumor-resected patient.

went FOLFIRI + BV after FOLFIRI alone. Similarly, there was no difference in the serum iron levels after the treatment between FOLFIRI alone $(205.09 \pm 139.37 \mu \mathrm{g} / \mathrm{dl}, \mathrm{n}=5)$ and FOLFIRI + BV $(257.45 \pm 151.63 \mu \mathrm{g} / \mathrm{dl})$.

Changes in transferrin and ferritin levels during FOLFOX4 and FOLFIRI therapies. The influence of chemotherapy on transferrin (an iron-transporting protein) (10) and ferritin (an iron storage protein) $(11,12)$ was also investigated. In the 15 patients of the FOLFOX4 group, transferrin levels were not different before $(256.79 \pm 80.13 \mathrm{mg} / \mathrm{dl})$ and after $(233.53 \pm 80.70 \mathrm{mg} / \mathrm{dl})$ the treatment $(\mathrm{p}=0.14)$. In the 14 patients of the FOLFOLI group, the transferrin level was $236.15 \pm 54.31 \mathrm{mg} / \mathrm{dl}$ before the treatment and decreased slightly to $196.50 \pm 36.85 \mathrm{mg} / \mathrm{dl}$ after the treatment $(\mathrm{p}<0.001)$, but these changes were within the normal range of serum transferrin.

In the 15 patients of the FOLFOX4 group, the ferritin levels were not different before $(192.32 \pm 224.88 \mathrm{ng} / \mathrm{dl})$ and after $(210.15 \pm 210.16 \mathrm{ng} / \mathrm{dl})$ the treatment $(\mathrm{p}=0.67)$. Similarly, in the 14 patients of the FOLFIRI group, the ferritin levels were not different before $(211.48 \pm 181.83 \mathrm{ng} / \mathrm{dl})$ and after $(220.15 \pm 182.97 \mathrm{ng} / \mathrm{dl})$ the treatment $(\mathrm{p}=0.83)$. These changes were all within the normal range of serum ferritin. 
Urinary iron excretion during FOLFOX4 and FOLFIRI therapies. To determine whether the changes in serum iron during chemotherapy were related to the urinary iron excretion, urine samples were collected on the day of treatment and on the next day to measure the urinary iron level in 5 and 7 patients of the FOLFOX4 and FOLFIRI groups, respectively. Although urinary iron excretion was $0.09 \mathrm{mg} /$ day on the day of treatment in 1 subject receiving FOLFIRI, it was within the normal range $(<0.2 \mathrm{mg} /$ day). Moreover, urinary iron excretion was below the detection limit $(0.03 \mathrm{mg} / \mathrm{day})$ in all of the other subjects.

\section{Discussion}

Recently, a powerful and effective combination chemotherapy has become available due to the development of antitumor chemotherapeutical agents and molecular targeting drugs. However, the incidence of serious adverse reactions has also increased. Almost all anticancer agents have the potential to induce myelosuppression by eliciting the apoptosis/necrosis of immature myelopoietic cells. In particular, severe leukopenia, thrombocytopenia and erythropenia are serious adverse events that lead to the termination of treatment. FOLFOX4 and FOLFIRI therapies are standard treatments for advanced colorectal cancer; however, they cause characteristic adverse reactions, such as peripheral neuropathy and severe diarrhea as well as conventional reactions like myelosuppression $(13,14)$.

During our preliminary studies on the adverse events caused by FOLFOX4 or FOLFIRI therapy, an increase in the serum iron level was sometimes observed, while the red blood cell count remained unchanged. Focusing on this finding, the present study was carried out.

In regard to the chemotherapy-induced changes in the serum iron level, Follézou et al reported that serum iron levels transiently increased during chemotherapy (9). However, their study differed from the present investigation in the following respects. Their patients received relatively older anticancer drugs, such as 5-FU, adriacin, and cyclophosphamide, and they did not measure the levels of transferrin or ferritin. Furthermore, they did not evaluate the effect of tumor cell death and hepatic damage on the increase in serum iron levels.

In the present study, we measured serum iron as well as transferrin and ferritin levels in patients who received FOLFOX4 or FOLFIRI therapy alone or in combination with BV. The serum iron level showed a transient increase in patients receiving FOLFOX4 or FOLFIRI therapy alone. In most of the patients, serum iron increased above the normal range (60-210 $\mu \mathrm{g} / \mathrm{dl}$ for men and 50-170 $\mu \mathrm{g} / \mathrm{dl}$ for women) and sometimes reached $400 \mu \mathrm{g} / \mathrm{dl}$. We confirmed that the serum iron level similarly increased regardless of the administration of $\mathrm{BV}$, suggesting that the transient increase in serum iron was not due to BV, but was presumably caused by FOLFOX4 or FOLFIRI therapy alone. In contrast, transferrin levels were not essentially changed during chemotherapy with FOLFOX4 or FOLFIRI. Moreover, ferritin levels were not basically changed by FOLFOX4 and FOLFIRI therapies.

Levels of aspartate aminotransferase, alanine aminotransferase and hemoglobin did not change during chemotherapy (data not shown). In addition, urinary excretion of iron was not increased by the chemotherapy. These observations suggest that the transient increase in serum iron was not due to the destruction of hepatocytes or erythrocytes. However, it is possible that iron was transiently released from tumor cells into the blood by chemotherapy. To examine this possibility, FOLFIRI therapy was administered to a patient after tumor resection (1 patient and 6 blood samples), and serum iron levels were measured (Fig. 7). As a result, it was revealed that the serum iron level increased transiently after FOLFIRI therapy even in the patient who had undergone tumor resection. Since a transient increase in serum iron level was also observed after tumor resection $(56.50 \pm 5.32 \mu \mathrm{g} / \mathrm{dl}$ before versus $239.0 \pm 18.95 \mu \mathrm{g} / \mathrm{dl}$ after the chemotherapy; an increase of $427.25 \pm 61.62 \%$ ), the increased iron was unlikely derived from tumor cells but was likely derived from normal cells. Thus, it is reasonable to speculate that the increased iron in sera was mainly derived from normal cells, since the number of normal cells was much higher than that of the tumor cells in the body. However, it cannot be ruled out that iron is partially released from tumor cells into the blood during chemotherapy in cancer-bearing patients.

If the transient increase in serum iron observed in the present study can estimate the outcome of FOLFOX4 or FOLFIRI therapy, it could be used as one of the potential biomarkers for monitoring antitumor chemotherapy. In fact, our preliminary studies revealed that the efficacy of FOLFOX4 or FOLFIRI therapy is correlated with the response of serum iron. We are now planning to investigate the relationship between the changes in serum iron and the outcome of chemotherapy in a larger polulation of patients.

\section{Acknowledgements}

We would like to thank the surgeons and the other medical staff at Tobu Chiiki Hospital for their cooperation.

\section{References}

1. De Gramont A, Figer A, Seymour M, et al: Leucovorin and fluorouracil with or without oxaliplatin as first-line treatment in advanced colorectal cancer. J Clin Oncol 18: 2938-2947, 2000.

2. Rougier P, Cutsem EV, Bajetta E, et al: Randomised trial of irinotecan versus fluorouracil by continuous infusion after fluorouracil failure in patients with metastatic colorectal cancer. Lancet 352: 1407-1412, 1998.

3. Grothey A and Sargent D: Overall survival of patients with advanced colorectal cancer correlates with availability of fluorouracil, irinotecan, and oxaliplatin regardless of whether doublet or single-agent therapy is used first line. J Clin Oncol 23: 9441-9442, 2005.

4. Giantonio BJ, Catalano PJ, Meropol NJ, et al: Bevacizumab in combination with oxaliplatin, fluorouracil, and leucovorin (FOLFOX4) for previously treated metastatic colorectal cancer: Results from the Eastern Cooperative Oncology Group study E3200. J Clin Oncol 25: 1539-1544, 2007.

5. Hurwitz H, Fehrenbacher L, Novotny W, et al: Bevacizumab plus irinotecan, fluorouracil, and leucovorin for metastatic colorectal cancer. N Engl J Med 350: 2335-2342, 2004.

6. Cunningham D, Humblet Y, Siena S, et al: Cetuximab monotherapy and cetuximab plus irinotecan in irinotecan-refractory metastatic colorectal cancer. N Engl J Med 351: 337-345, 2004.

7. Saltz LB, Lenz H-J, Kindler HL, et al: Randomized Phase II trial of cetuximab, bevacizumab, and irinotecan compared with cetuximab and bevacizumab alone in irinotecan-refractory colorectal cancer: The BOND-2 study. J Clin Oncol 25: 4557-4561, 2007. 
8. Cutsem EV, Kohne C-H, Hitre E, et al: Cetuximab and chemotherapy as initial treatment for metastatic colorectal cancer. $\mathrm{N}$ Engl J Med 360: 1408-1417, 2009.

9. Follézou JY and Bizon M: Cancer chemotherapy induces a transient increase of serum-iron level. Neoplasma 33: 225-231, 1986.

10. Hentze MW, Muckenthaler MU, Andrews NC, et al: Molecular control of mammalian iron metabolism. Cell 117: 285-297, 2004.

11. Iwai $\mathrm{K}$ : An ubiquitin ligase recognizing a protein oxidized by iron: implications for the turnover of oxidatively damaged proteins. J Biochem 134: 175-182, 2003.
12. Rouault TA: The role of iron regulatory proteins in mammalian iron homeostasis and disease. Nat Chem Biol 2: 406-414, 2006.

13. Goldberg RM, Sargent DJ, Morton RF, et al: A randomized controlled trial of fluorouracil plus leucovorin, irinotecan, and oxaliplatin combinations in patients with previously untreated metastatic colorectal cancer. J Clin Oncol 22: 23-30, 2004.

14. Douillard JY, Cunningham D, Roth AD, et al: Irinotecan combined with fluorouracil compared with fluorouracil alone as first-line treatment for metastatic colorectal cancer: a multicentre randomised trial. Lancet 355: 1041-1047, 2000. 